\title{
Use quantitative parameters in spectral computed tomography for the differential diagnosis of metastatic mediastinal lymph nodes in lung cancer patients
}

\author{
Suidan Huang, Hongjia Meng, Renli Cen, Zhiwen Ni, Xiaoling Li, Sushant Suwal, Huai Chen \\ Department of Radiology, First Affiliated Hospital of Guangzhou Medical University, Guangzhou, China \\ Contributions: (I) Conception and design: H Chen, S Huang; (II) Administrative support: H Chen; (III) Provision of study materials or patients: R \\ Cen, H Meng; (III) Collection and assembly of data: S Huang, Z Ni; (IV) Data analysis and interpretation: Z Ni, H Meng, S Suwal; (V) Manuscript \\ writing: All authors; (VI) Final approval of manuscript: All authors. \\ Correspondence to: Huai Chen. Department of Radiology, First Affiliated Hospital of Guangzhou Medical University, Guangzhou 510120, China. \\ Email: chenhuai1977@163.com.
}

\begin{abstract}
Background: Accurate diagnosis of mediastinal lymph node (LN) metastases is very important for the treatment and prognosis in lung cancer patients. Spectral computed tomography (CT), as a non-invasive approach, has good prospects for detecting mediastinal nodal metastasis. However, the diagnostic criteria of differentiating metastatic and nonmetastatic LNs have not been determined.

Methods: Clinical and imaging data of 64 lung cancer patients (mean age $61.3 \pm 10.3$ years, 41 men) from April to December 2019 were retrospectively analyzed. The unenhanced scan and contrast enhanced arterial phase (AP) and venous phase (VP) spectral CT scans were performed. The $70 \mathrm{keV}$ monochromatic image and iodine-based image in all phases were analyzed to measure the parameters of LNs. LNs were divided into the metastatic and non-metastatic groups based on confirmative pathological results, and their differences were statistically analyzed. The receiver operating characteristics curve (ROC) was used to evaluate the efficacy of the differential diagnosis.

Results: Seventy-four metastatic LNs and 152 non-metastatic LNs were obtained. Compared with nonmetastatic LNs, metastatic LNs often had a larger size $(\mathrm{P}<0.001)$. In the unenhanced scans, the density of metastatic LNs was lower than that of non-metastatic LNs $(\mathrm{P}<0.001)$; however, there was no difference in CT value in $\mathrm{AP}$ and VP between metastatic and non-metastatic $\mathrm{LNs}(\mathrm{P}=0.07, \mathrm{P}=0.08$, respectively). A statistically significant difference was found in iodine concentration (IC), normalized iodine concentration (NIC) and slope of the spectral curve $(\lambda \mathrm{HU})$ in unenhanced scan, IC and $\lambda \mathrm{HU}$ in $\mathrm{AP}$, as well as IC, NIC and $\lambda \mathrm{HU}$ in VP between metastatic and non-metastatic LNs. There was no difference in NIC in AP between them.

Conclusions: Combined with morphology, spectral CT quantitative parameters demonstrate certain diagnostic efficiency for differential diagnosis between metastatic and non-metastatic LNs in lung cancer patients.
\end{abstract}

Keywords: Lymph node metastasis; lung cancer; spectral computed tomography (spectral CT)

Submitted Mar 08, 2021. Accepted for publication Jun 23, 2021.

doi: $10.21037 /$ jtd-21-385

View this article at: https://dx.doi.org/10.21037/jtd-21-385

\section{Introduction}

Lung cancer is the malignant tumor with the highest morbidity and mortality currently. It is reported that the 5 -year survival rate of non-small cell lung cancer (NSCLC) is about $23.6 \%$, while that of small cell lung cancer (SCLC) is only $7 \%$ (1). Among the patients with lymph node (LN) metastasis, only $26 \%$ to $53 \%$ of patients had a survival of more than 5 years $(2,3)$. Accurate staging of $\mathrm{LN}$ metastases 
is very important for the treatment and prognosis of lung cancer patients.

Computed tomography (CT) is commonly used to evaluate the mediastinal LN metastasis in clinical practice. Short diameter of a lesion larger than $1 \mathrm{~cm}$ is usually used as the diagnostic criteria for LN metastasis, but the overall accuracy is not high. Almeida et al. (4) studied the diagnostic criteria and found that CT yielded a sensitivity and specificity of $51 \%$ and $85 \%$, respectively. Nguyen et al. found that the false positive rate and false negative rate were $40 \%$ and $20 \%$, respectively (5). Positron emission tomography (PET)/CT can also be used to diagnose mediastinal LN metastasis of lung cancer, but the main limitations of PET/CT are a high radiation exposure, limited availability and high economic demands (6). Zhao et al. (7) performed a meta-analysis to evaluate the role of F-18 FDG PET/CT in detecting mediastinal nodal metastasis and showed a $72 \%$ sensitivity and $89 \%$ specificity. But Zhai et al. (8) indicated that, in the diagnosis of metastatic and non-metastatic LNs, the threshold of SUVmax of LN is still controversial. A meta-analysis study in (9) showed that pooled specificity for F-18 FDG PET or PET/CT was 0.65 [95\% confidence interval (CI): $0.57-$ $0.72]$, meaning that false positive rate was $35 \%$. Hence, at present, PET/CT is still not routinely used in every patient in clinic. LN biopsy is the gold standard for diagnosis, but the invasive procedure can increase the discomfort of patients and the risk of complications (10). Thus, new methods, especially non-invasive approaches, for detecting mediastinal nodal metastasis in lung cancer are needed.

Characterized by instantaneous double KVp switching, spectral CT imaging can obtain spectral curve slope and the separation image of substances, so as to identify substances with different chemical composition (11-13). The main difference between spectral and traditional CT is that the latter can only depict the changes of different substances through density. Sun et al. (14) indicated that traditional CT is not able to distinguish two different substances with the same density, but spectral CT can solve this problem. At present, spectral CT has been used to determine LN metastasis in multiple tumors such as rectal cancer, gastric cancer and laryngeal cancer (15-17), and these studies have shown that spectral CT has a certain diagnostic ability in $\mathrm{LN}$ characterization. However, there lacks universal guidelines for using spectral CT in the quantitative determination of mediastinal LNs in lung cancer. The purpose of this study was to explore using quantitative parameters in spectral CT for diagnosing mediastinal LN metastases in lung cancer patients.

We present the following article in accordance with the STROBE reporting checklist (available at https://dx.doi. org/10.21037/jtd-21-385).

\section{Methods}

\section{Subjects}

The study was conducted in accordance with the Declaration of Helsinki (as revised in 2013). The study was approved by the Ethics Committee of First Affiliated Hospital of Guangzhou Medical University (Medical research ethical review 2019, No. K47) and individual consent for this retrospective analysis was waived. Sixty-four lung cancer patients admitted to our hospital from April to December 2019 were selected in this study (Figure 1). All the patients underwent unenhanced chest CT scan and contrast enhanced arterial phase (AP) and venous phase (VP) spectral CT scans. The primary lesion and mediastinal LNs were proven pathologically by operation or puncture, including 55 patients who underwent resection of lung cancer and $\mathrm{LN}$ dissection, and nine patients who underwent bronchoscopic LN biopsy. There were 31 cases with $\mathrm{LN}$ metastasis and 33 cases without $\mathrm{LN}$ metastasis. Our inclusion criterion is that the short diameter of LNs must be larger than $5 \mathrm{~mm}$ (18). Because it is technically challenging to position the circular region of interest (ROI) in these small LNs that are less than $5 \mathrm{~mm}$, we chose LNs whose short diameters were larger than $5 \mathrm{~mm}$. Patients who had chemotherapy, radiotherapy, and target therapy were excluded. Patients whose images had artifacts that affects measurement or location of LNs on CT was unclear were also excluded. Among the 64 patients, there were 41 males and 23 females, ranging in age from 33 to 87 years old, with an average age of $61.3 \pm 10.3$ years. The primary pathological types of 64 lung cancer patients are shown in Table 1. Adenocarcinoma is the most common (37/64), followed by squamous cell carcinoma (10/64).

\section{Image acquisition}

GE Revolution 256 row energy spectrum CT machine was used. All patients were scanned with Gemstone Spectral Imaging (GSI) mode. The scanning range was from the thoracic entrance to the level of costal diaphragmatic angle. Energy spectrum GSI scanning was performed in unenhanced scan, AP and VP, and the voltage was switched 


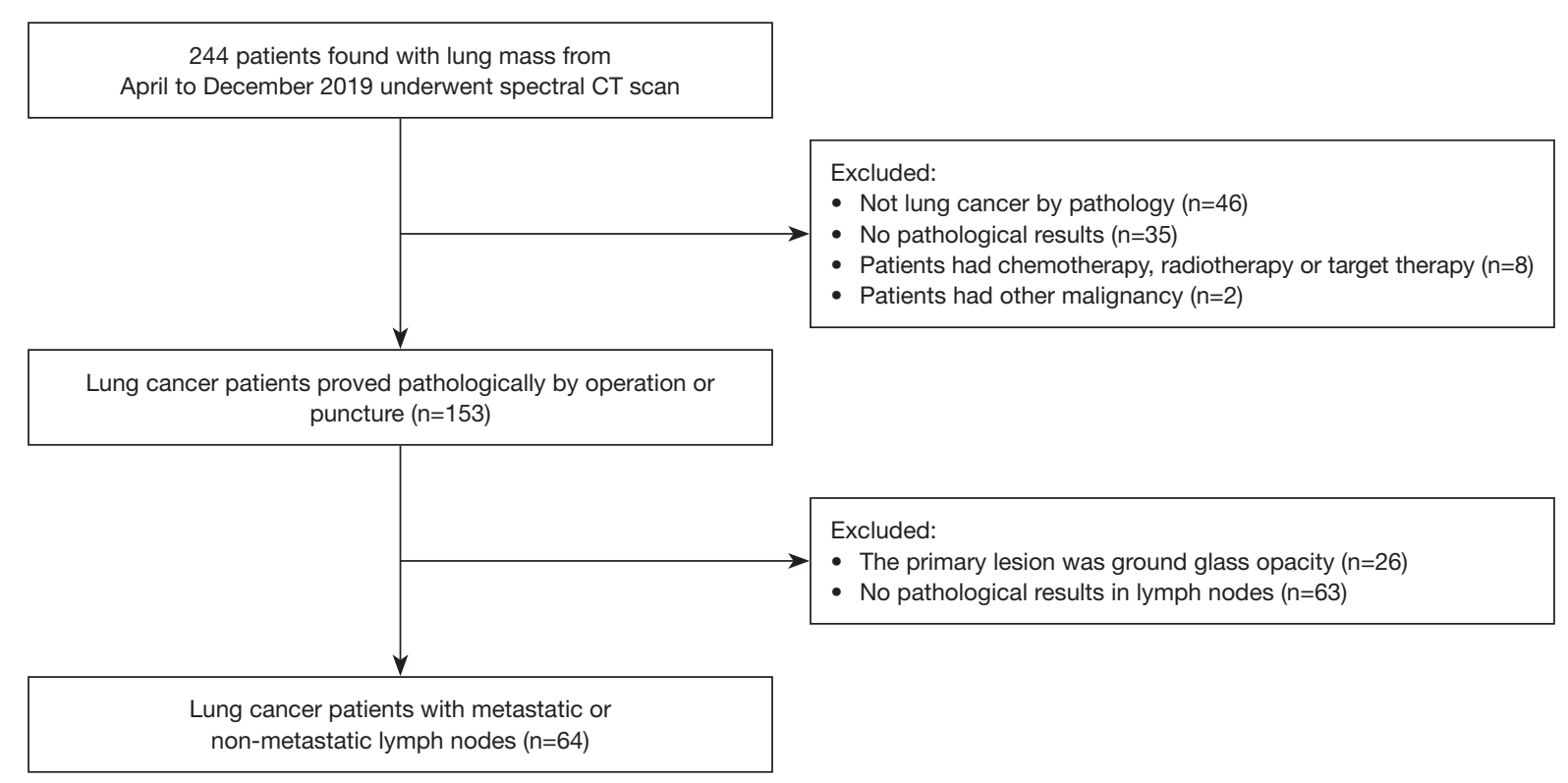

Figure 1 The inclusion and exclusion of patients.

Table 1 Clinical data of 64 patients with lung cancer

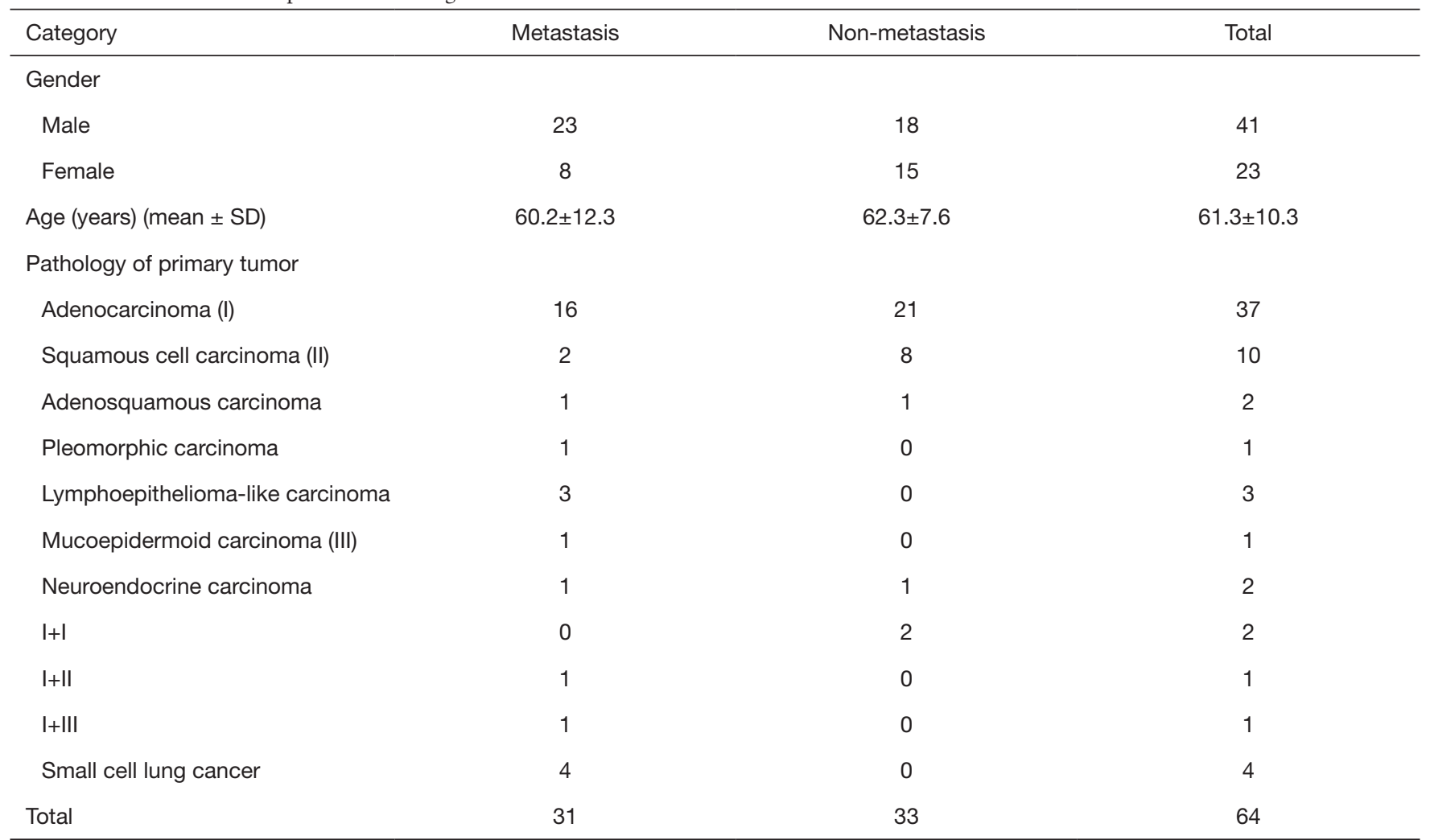

I+I means patient had two primary tumors, which were both adenocarcinomas. I+II means patient had both Adenocarcinoma and Squamous cell carcinoma. I+III means patient had both Adenocarcinoma and Mucoepidermoid carcinoma. SD, standard deviation. 
between high and low energy ( 80 and $140 \mathrm{kVp})(0.25 \mathrm{~s}$ ). A non-ionic contrast agent, iodixanol $(320 \mathrm{mg} \mathrm{I} / \mathrm{mL})$, was injected with a double-cylinder high-pressure syringe through the anterior cubital venous group, with a flow rate of $4.5 \mathrm{~mL} / \mathrm{s}$ and $1.0 \mathrm{mg} / \mathrm{kg}$. AP was scanned $22 \mathrm{~s}$ after injection of contrast agent, and VP was scanned $60 \mathrm{~s}$ later. The scanning parameters were as follows: spiral scanning mode, frame rotation time $0.5 \mathrm{~s}$ rotation, pitch 0.992 , tube current: automatic millisecond, layer thickness and interval $0.625 \mathrm{~mm}$, and field of view (FOV) $350 \mathrm{~mm} \times 350 \mathrm{~mm}$.

\section{CT image analysis}

All the original images were uploaded to an GE ADW4.7 post-processing and reconstruction workstation with $1.25-\mathrm{mm}$ thickness. The $70-\mathrm{keV}$ single-energy image with the highest signal-to-noise ratio in monoenergetic mode was selected to measure the short diameter of LNs, the $\mathrm{CT}$ values of single-energy $70 \mathrm{keV}$ in unenhanced scan, and AP and VP scans. And the iodine concentration (IC, $100 \mu \mathrm{g} / \mathrm{cm}^{3}$ ), water concentration (WC) and aortic iodine concentration (ICaorta) were measured. The normalized iodine concentration (NIC) was calculated as the ratio of IC divided by ICaorta, i.e., NIC = IC/ ICaorta. After drawing the ROI around each target object, the computer automatically obtained the CT value under different single energy to form the energy spectrum attenuation curve. The CT value of 40 and $140 \mathrm{keV}$ of each spectral image were measured, which was used to calculate the slope of the spectrum curve $(\lambda \mathrm{HU})$. The $\lambda \mathrm{HU}$ was calculated, with 40 and $100 \mathrm{keV}$ serving as the reference points, by dividing the difference in $\mathrm{CT}$ value by the difference in energy between the two points, that is, $\lambda \mathrm{HU}=\left(\mathrm{CT}_{40 \mathrm{keV}}-\mathrm{CT}_{100 \mathrm{keV}}\right) / 60(19)$.

ROI around LN was placed on the slices showing the maximum cross-section of the legions. The placement of ROI was to avoid surrounding fat tissue, necrosis, calcification, and blood vessels. In the meantime, we tried to place the ROIs in a consistent manner in terms of size, shape, location and on the same slices across the unenhanced scan, and AP and VP scans.

\section{Imaging and pathology comparison criteria}

Mediastinal LNs were divided according to the map of the 2009 International Association for the Study of Lung Cancer (IASLC map) $(20,21)$, and compared with the postoperative or puncture pathological results of the corresponding regional LNs. The selection of LNs was based on surgical record and pathological result. In this process, we made a one-to-one match between each $\mathrm{LN}$ on the CT and the pathological confirmation and discarded the LNs that could not be matched with pathology.

\section{Statistical analysis}

We used SPSS 25.0 software for statistical analysis. For the comparison of two independent groups of measurement data, if each group of data obeys normal distribution, then independent sample $t$-test was used, otherwise the rank sum test (Wilcoxon) was used. Receiver operating characteristics (ROC) curve was created for the measurement data with statistically significant parameters between the two groups to determine the optimal cut-off value. Sensitivity, specificity, and the area under the curve (AUC) were calculated. All the parameters that had significant differences, including spectral and traditional CT parameters, were analyzed in combination. A test level $\mathrm{P}<0.05$ was considered statistically significant.

\section{Results}

\section{Data of the subjects}

The primary pathological types of 64 lung cancer patients are shown in Table 1. There were 31 cases with LN metastasis and 33 cases without metastasis. A total of 74 metastatic and 152 non-metastatic LNs were obtained, and 22 LNs were excluded due to artifacts. The zones of metastatic and nonmetastatic LNs are shown in Table 2.

\section{Quantitative parameter analysis of metastatic and non-metastatic LNs}

\section{Short diameter and CT value of LNs}

The slice which showed the largest cross section of a LN was selected for measurement. The short diameter of metastatic LNs ranges from 7.1 to $44.0 \mathrm{~mm}$, with an average of $14.35 \pm 7.73 \mathrm{~mm}$. The short diameter of nonmetastatic LNs ranges from 5.0 to $13.9 \mathrm{~mm}$, with an average of $8.18 \pm 1.77 \mathrm{~mm}$. Statistically, the short diameter of metastatic LNs is larger than that of non-metastatic LNs $(\mathrm{P}<0.001)$ (Table 3). Of the 74 metastatic LNs, 48 (64.9\%) had a short diameter $\geq 10 \mathrm{~mm}$, while $26(35.1 \%)$ had a short diameter $<10 \mathrm{~mm}$. Of 152 non-metastatic LNs, 128 (84.2\%) had a short diameter $<10 \mathrm{~mm}$, but $24(15.8 \%)$ had a short 
Table 2 Distribution of 226 lymph nodes

\begin{tabular}{lcc}
\hline Zones & $\begin{array}{c}\text { Metastatic lymph nodes } \\
(\mathrm{n}=74), \mathrm{n}(\%)\end{array}$ & $\begin{array}{c}\text { Non-metastatic lymph } \\
\text { nodes }(\mathrm{n}=152), \mathrm{n}(\%)\end{array}$ \\
\hline 1 & $0(0.0)$ & $0(0.0)$ \\
2 & $6(8.1)$ & $15(9.9)$ \\
3 & $0(0.0)$ & $0(0.0)$ \\
4 & $19(25.7)$ & $32(21.1)$ \\
5 & $9(12.2)$ & $13(8.6)$ \\
6 & $0(0.0)$ & $0(0.0)$ \\
7 & $16(21.6)$ & $42(27.6)$ \\
8 & $1(1.4)$ & $0(0.0)$ \\
9 & $0(0.0)$ & $2(1.3)$ \\
10 & $8(10.8)$ & $32(21.1)$ \\
11 & $14(18.9)$ & $16(10.5)$ \\
12 & $1(1.4)$ & $0(0.0)$ \\
\hline
\end{tabular}

diameter $\geq 10 \mathrm{~mm}$.

In terms of CT value (Table 3), the unenhanced scan of metastatic LN was about $39.00 \pm 9.33 \mathrm{HU}$, and that of nonmetastatic LN was $45.20 \pm 12.83 \mathrm{HU}$. There was significant difference in unenhanced scan CT value between metastatic and non-metastatic LNs $(\mathrm{P}<0.001)$, but there was no significant difference between $\mathrm{AP}$ and $\mathrm{VP}$ scans $(\mathrm{P}=0.07$, 0.076 , respectively).

\section{Quantitative parameter of spectral CT}

There were significant differences in IC, NIC and $\lambda \mathrm{HU}$ between metastatic $\mathrm{LNs}$ and non-metastatic LNs on unenhanced scan $(\mathrm{P}<0.001, \mathrm{P}=0.007, \mathrm{P}<0.001)$ (Table 4). The IC, NIC and $\lambda \mathrm{HU}$ of non-metastatic LNs were higher than those of metastatic LNs (Figures 2,3), but there was no significant difference in WC between metastatic and nonmetastatic LNs. In AP, the IC and $\lambda \mathrm{HU}$ of non-metastatic

Table 3 Comparison of short diameter and CT value of metastatic and non-metastatic LNs

\begin{tabular}{|c|c|c|c|c|c|c|c|c|}
\hline Variables & Metastatic LNs & Non-metastatic LNs & $P$ value & AUC & $95 \% \mathrm{Cl}$ & Critical value & Sensitivity (\%) & Specificity (\%) \\
\hline Short diameter $(\mathrm{mm})$ & $14.35 \pm 7.73$ & $8.18 \pm 1.77$ & $<0.001$ & 0.853 & $0.803,0.904$ & 10.75 & 60.8 & 92.8 \\
\hline $\mathrm{NC}-\mathrm{CT}(\mathrm{HU})$ & $39.00 \pm 9.33$ & $45.20 \pm 12.83$ & $<0.001$ & 0.637 & $0.564,0.709$ & 48.95 & 87.8 & 38.8 \\
\hline AP-CT (HU) & $63.30 \pm 19.42$ & $67.71 \pm 19.30$ & 0.070 & - & - & - & - & - \\
\hline VP-CT (HU) & $77.17 \pm 15.99$ & $81.59 \pm 20.29$ & 0.076 & - & - & - & - & - \\
\hline
\end{tabular}

$\mathrm{AP}$, arterial phase; AUC, area under the curve; Cl, confidence interval; CT, computed tomography; LNs, lymph nodes; NC, non-contrast; $\mathrm{VP}$, venous phase.

Table 4 Quantitative parameters of spectral CT between metastatic and non-metastatic LNs

\begin{tabular}{|c|c|c|c|c|c|c|c|c|}
\hline Variables & Metastatic LNs & Non-metastatic LNs & $P$ value & AUC & $95 \% \mathrm{Cl}$ & Critical value & Sensitivity (\%) & Specificity (\%) \\
\hline NC-IC & $7.07 \pm 2.36$ & $8.85 \pm 2.54$ & $<0.001$ & 0.697 & $0.624,0.769$ & 6.83 & 54.1 & 77.0 \\
\hline NC-NIC & $1.56(1.19,2.01)$ & $1.81(1.37,2.42)$ & 0.007 & 0.611 & $0.534,0.689$ & 1.69 & 64.9 & 57.2 \\
\hline $\mathrm{NC}-\lambda \mathrm{HU}$ & $0.80(0.63,1.08)$ & $1.05(0.86,1.29)$ & $<0.001$ & 0.701 & $0.628,0.774$ & 0.87 & 56.8 & 75.0 \\
\hline AP-IC & $18.84 \pm 8.03$ & $23.51 \pm 7.66$ & $<0.001$ & 0.671 & $0.595,0.747$ & 18.55 & 52.7 & 73.7 \\
\hline AP-WC & $1,011.73 \pm 13.41$ & $1,004.81 \pm 13.77$ & $<0.001$ & 0.672 & $0.598,0.747$ & $1,009.61$ & 64.9 & 65.1 \\
\hline $\mathrm{AP}-\lambda \mathrm{HU}$ & $2.22(1.54,2.76)$ & $2.70(2.22,3.29)$ & $<0.001$ & 0.679 & $0.604,0.754$ & 2.10 & 47.3 & 81.6 \\
\hline VP-IC & $22.61 \pm 5.89$ & $25.47 \pm 5.44$ & 0.004 & 0.618 & $0.540,0.697$ & 18.74 & 27.0 & 91.4 \\
\hline VP-NIC & $0.51(0.43,0.56)$ & $0.53(0.46,0.62)$ & 0.016 & 0.599 & $0.522,0.676$ & 0.59 & 85.1 & 32.9 \\
\hline
\end{tabular}

$\mathrm{AP}$, arterial phase; AUC, area under the curve; $\mathrm{Cl}$, confidence interval; CT, computed tomography; IC, iodine concentration (100 $\left.\mu \mathrm{g} / \mathrm{cm}^{3}\right)$; LNs, lymph nodes; NC, non-contrast; NIC, standardized iodine concentration; VP, venous phase; WC, water concentration (mg/cm³); $\lambda \mathrm{HU}^{3}$, slope of the spectral curve. 

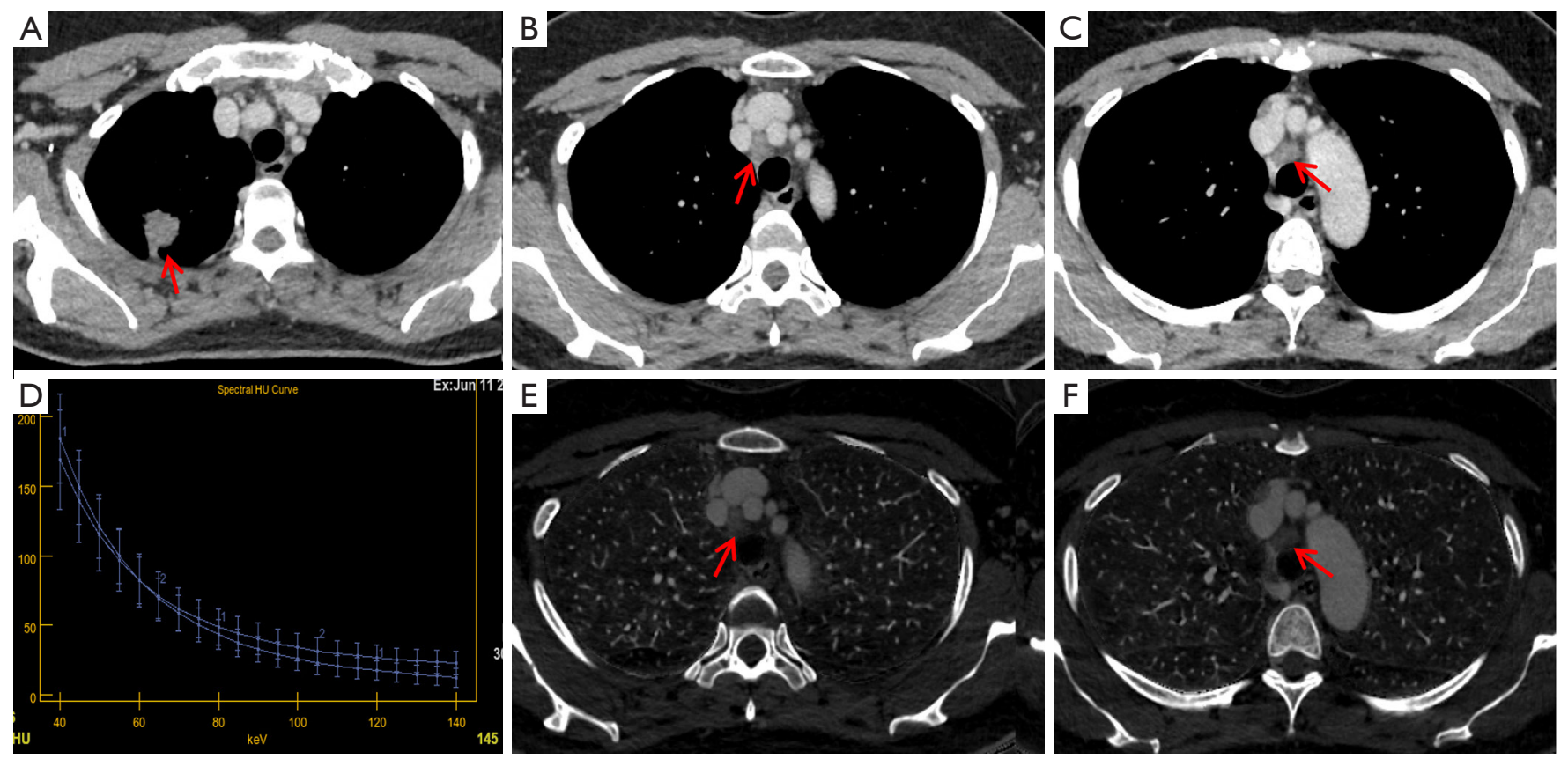

Figure 2 Infiltrative adenocarcinoma of right upper lung with 2R and 4R LN metastases. (A-C) The VP 70-KeV monochromatic images showed the primary tumor (A, red arrow), 2R LN metastasis (B, red arrow), and 4R LN metastasis (C, red arrow), and the CT value of $2 \mathrm{R}$ and $4 \mathrm{R} \mathrm{LN}$ metastases was 63.6 and 61.2 HU. (D) The $\lambda H U$ of $2 \mathrm{R}$ and 4R LN metastases in VP were 2.62 and 2.23. (E,F) In iodine-based images, IC and NIC of 2R (E, red arrow), and 4R (F, red arrow) LN metastases were $23.10\left(100 \mu \mathrm{g} / \mathrm{cm}^{3}\right), 0.47$ and $19.82\left(100 \mu \mathrm{g} / \mathrm{cm}^{3}\right), 0.40$, respectively. CT, computed tomography; IC, iodine concentration; LN, lymph node; NIC, normalized iodine concentration; VP, venous phase; $\lambda H U$, slope of the spectral curve.

LNs were higher than those of metastatic LNs $(\mathrm{P}<0.001$, $\mathrm{P}<0.001, \mathrm{P}<0.001$, respectively). The $\mathrm{WC}$ in $\mathrm{AP}$ of nonmetastatic LNs was also significantly higher than that of metastatic LNs $(\mathrm{P}<0.001)$, but there was no significant difference in NIC between the two groups $(\mathrm{P}=0.092)$. In VP, IC, NIC and $\lambda \mathrm{HU}$ of non-metastatic LNs were significantly higher than those of the metastatic $\mathrm{LNs}(\mathrm{P}=0.004, \mathrm{P}=0.016$, $\mathrm{P}=0.003$, respectively). However, there was no statistical significance in $\mathrm{WC}(\mathrm{P}=0.05)$.

\section{ROC curve analysis of metastatic and non-metastatic LNs}

The statistically significant parameters were analyzed by ROC curve (Table 4). The largest AUC was the short diameter of LNs, AUC =0.853 (95\% CI: 0.803-0.904). When the Jordan index reached its maximum, the cut-off value was $10.75 \mathrm{~mm}$ (sensitivity $60.8 \%$, specificity $92.8 \%$ ), the positive likelihood ratio (LR) (+) was 8.4 and negative LR (-) was 0.42 . The diagnosis value of spectral parameters combined with traditional CT parameters improved, with a combined AUC $=0.877$ (95\% CI: 0.829-0.952), sensitivity $82.4 \%$, and specificity $77.6 \%$.

\section{Discussion}

LN staging is very important as it affects the treatment and prognosis of patients with lung cancer $(22,23)$. In the case of excluding distant metastasis, it is crucial to determine whether there is mediastinal LN metastasis. However, accurate $\mathrm{N}$ staging in TNM staging is more difficult than $\mathrm{T}$ staging. It has been reported that the total coincidence rate of preoperative clinical TNM staging and postoperative pathological TNM staging in patients with lung cancer is only $39 \%$, mainly because of the low diagnostic accuracy in determining metastatic mediastinal LNs. At present, the commonly used diagnostic criteria for mediastinal LN metastasis of lung cancer is that the short diameter of LNs is more than $10 \mathrm{~mm}$. However, for metastatic LNs with a short diameter less than $10 \mathrm{~mm}$ and non-metastatic LNs larger than $10 \mathrm{~mm}$, the above criterion causes misdiagnosis. In our study, $26(35.1 \%)$ of 74 metastatic LNs had a short diameter $<10 \mathrm{~mm}$ and $24(15.8 \%)$ of 152 non-metastatic LNs had a short diameter $\geq 10 \mathrm{~mm}$ (Table S1), indicating a certain overlap in size between metastatic and nonmetastatic LNs. 

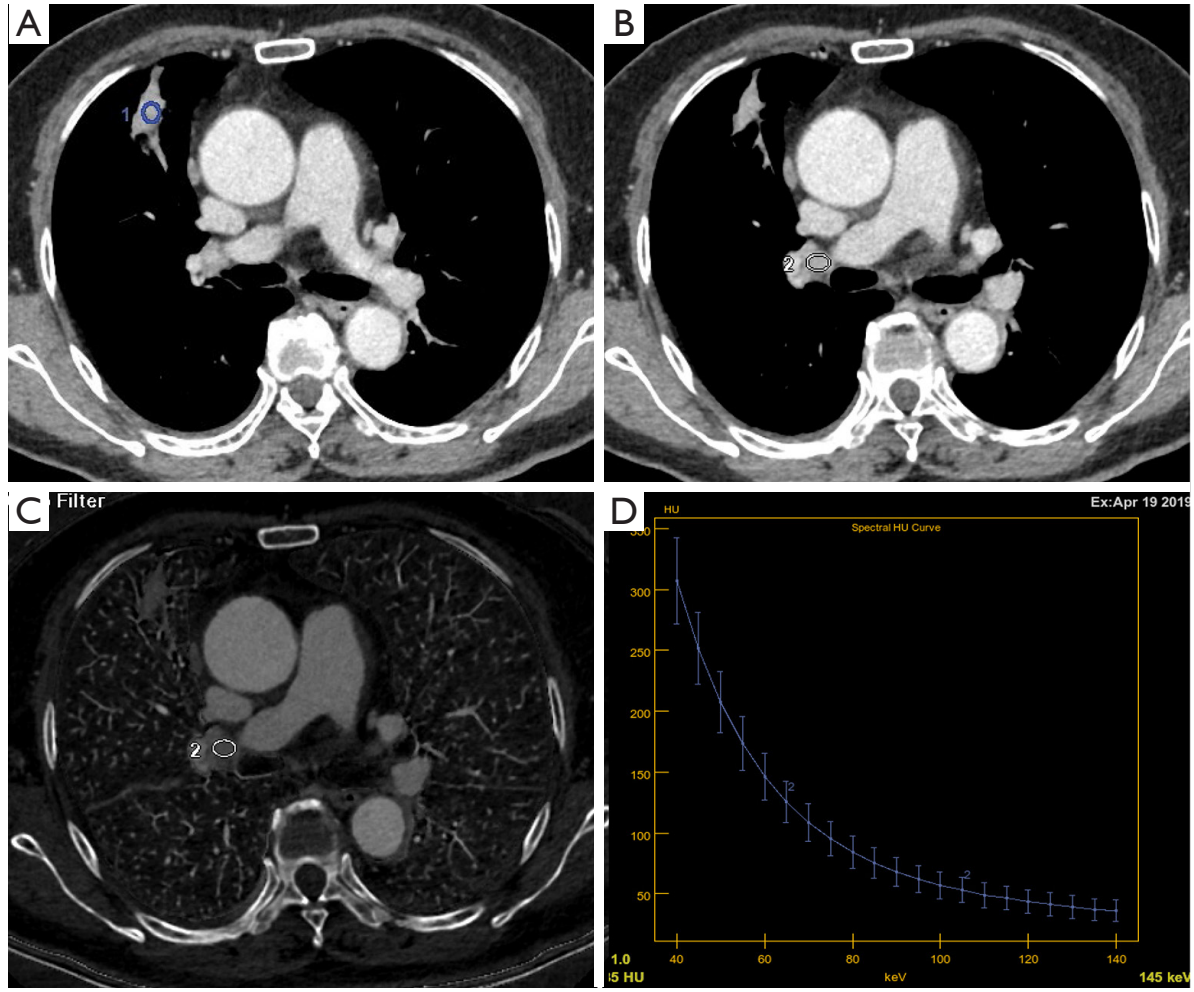

Figure 3 Infiltrative adenocarcinoma of right upper lung without right lung hilum (10R) LN metastasis. (A,B) The VP 70-KeV monochromatic images showed primary tumor (A) and 10R non-metastatic LN (B), and the CT value of 10R LN metastasis was 108.4 HU. (C) In iodine-based images, IC and NIC of $10 \mathrm{R} \mathrm{LN}$ were $34.39\left(100 \mu \mathrm{g} / \mathrm{cm}^{3}\right)$ and 0.52 . (D) In the spectral curve of $10 \mathrm{R} \mathrm{LN}, \lambda \mathrm{HU}$ was 4.25 . The blue and white circles represent the ROI we draw in the primary lesion and $10 \mathrm{R} \mathrm{LN}$ respectively. CT, computed tomography; IC, iodine concentration; LN, lymph node; NIC, normalized iodine concentration; VP, venous phase; $\lambda H U$, slope of the spectral curve; ROI, region of interest.

Spectral CT can distinguish lesions of different chemical components by energy spectrum attenuation curve (24). In this study, the $\lambda \mathrm{HU}$ of non-metastatic LNs was larger than that of metastatic LNs in three phases $(\mathrm{P}<0.001, \mathrm{P}<0.001$, and $\mathrm{P}=0.003)$, indicating that the spectral curve of nonmetastatic LNs was different from that of metastatic LNs. The energy spectrum curve of unenhanced scans has a higher diagnostic value in distinguishing between metastatic and non-metastatic LNs than enhanced scans. We considered that the slope of spectral curve of unenhanced scans accurately reflects the heterogeneity of the tissue composition. Intuitively, for metastatic LNs with tumor cell infiltration and normal LNs, their inner cell compositions are different, which may result in different spectral curves, that is, spectral curve can identify substances with different chemical composition. But in enhanced scans, the enhancement of each $\mathrm{LN}$ varies. We postulated that different concentration of iodine will affect how spectral curve identifies the underlying substances, and, as the result indicated, $\lambda \mathrm{HU}$ on unenhanced scans was more meaningful than enhanced scans.

Apfaltrer et al. and Chen et al. separately have shown that the quantification of iodine can be used as a marker of tumor vascular distribution $(25,26)$. In this study, the IC of metastatic LNs in AP and VP and NIC in VP were lower than those in non-metastatic LNs, which was consistent with the observation made by other researchers $(15,18,27,28)$, indicating that the vascular density of metastatic LNs was lower than that of non-metastatic LNs. On one hand, pathophysiological changes that affect tissue blood volume and vascular permeability will influence the degree of enhancement on CT. Although tumor cells produce vascular endothelial growth factor (VEGF) to stimulate neovascularization, using enhancement of blood vessels as a biomarker for metastases may not be robust as studies have shown the different types of lung cancer may have various degree of neovascularization and they have difference likelihoods of developing necrosis (29). In 

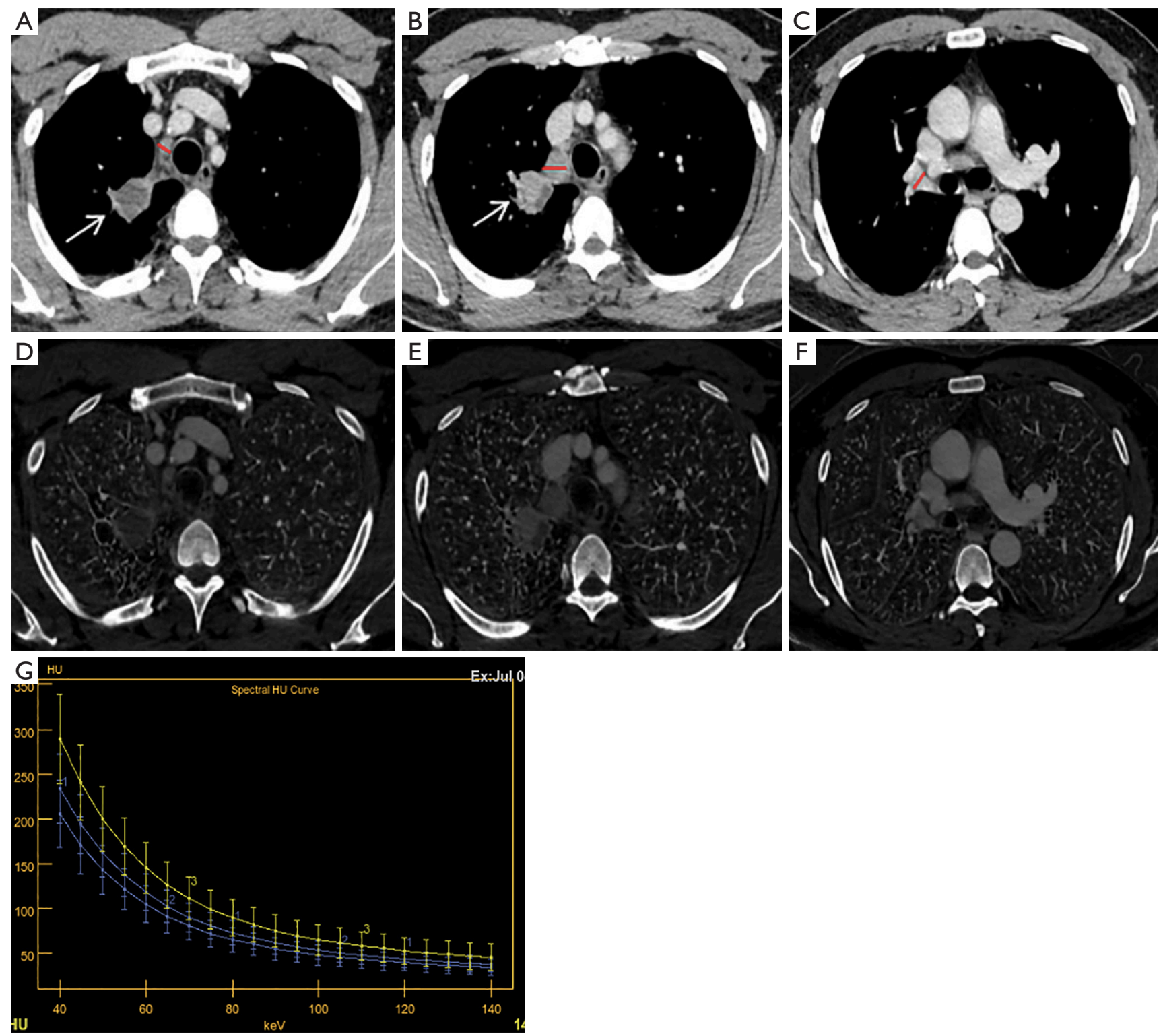

Figure 4 Lymphoepithelioma-like carcinoma of right upper lung with 2R and 4R LN metastases and without right hilum (10R) LN metastasis. (A-C) The VP 70-KeV monochromatic images showed primary tumor (white arrow) and 2R metastatic LNs (red line, A), 4 $\mathrm{R}$ metastatic LNs (red line, B), 10R non-metastatic LNs (red line, C). The short diameters and CT values of $2 \mathrm{R}, 4 \mathrm{R}$ and $10 \mathrm{R} \mathrm{LNs}$ were 8.3, 15.8, $11.5 \mathrm{~mm}$ and 68.7, 89, 105.2 HU, respectively. (D-F) In iodine-based images, IC and NIC of 2R, 4R and 10R LNs were 22.33 $\left(100 \mu \mathrm{g} / \mathrm{cm}^{3}\right), 0.46,24.92\left(100 \mu \mathrm{g} / \mathrm{cm}^{3}\right), 0.51$ and $30.89\left(100 \mu \mathrm{g} / \mathrm{cm}^{3}\right), 0.64$, respectively. (G) The spectral curve slope and $\lambda \mathrm{HU}$ of $2 \mathrm{R}$ (blue line 1), 4R (blue line 2) LN metastases and 10R (yellow line 3) non-metastatic LNs in VP were 2.73, 3.0, and 3.71, respectively. IC, iodine concentration; LN, lymph node; NIC, normalized iodine concentration; VP, venous phase; $\lambda \mathrm{HU}$, slope of the spectral curve.

addition, the infiltration of tumor cells may cause vascular stenosis or even occlusion, resulting in a low degree of enhancement. On the other hand, non-metastatic LNs have normal and mature vessels, giving them a higher degree of enhancement on CT. Indeed, previous studies have shown that the blood vessel density of non-metastatic LNs is higher than that of metastatic LNs (30), so the IC and NIC of metastatic LNs are lower than those of nonmetastatic LNs. Figure 4 showed that IC and NIC of $2 \mathrm{R}$ and $4 \mathrm{R}$ metastatic $\mathrm{LNs}$ are larger than that of $10 \mathrm{R}$ non- 
metastatic LNs. In terms of CT value, density of nonmetastatic LNs on unenhanced scans was generally higher than that of metastatic LNs $(\mathrm{P}<0.001)$, and the cut-off value was $48.95 \mathrm{HU}$, but the diagnostic efficacy $(\mathrm{AUC}=0.637$ ) and specificity $(38.8 \%)$ were not high, and there was no significant difference in CT values between the two groups measured in $\mathrm{AP}$ and $\mathrm{VP}$. Our results show that the iodine quantification in energy spectrum CT can better assess the blood supply and perfusion of tissue than CT value. As solid components of LNs were mainly involved in the measurement, calcification, fat, and blood vessels were not included in traditional CT. If there is no necrosis in some metastatic LNs, it is difficult to identify them as benign or malignant on traditional CT. Therefore, the iodine quantification in energy spectrum CT can better reflect the difference of tissue blood flow and perfusion between metastatic and non-metastatic LNs than CT value.

There was significant difference in NIC of the LNs in VP between the two groups, but there was no significant difference in NIC in AP. The calculation of NIC can exclude the influence of systemic circulation, blood vessels and other factors between different individuals, and can reflect the local blood supply more truly and objectively. However, due to the low degree of enhancement of LNs in the AP, the IC of the aorta varies greatly at different levels, affecting the stability of NIC. The distribution of contrast media in the LNs is more uniform and closer to the peak of enhancement in the VP, which can better reflect the outflow of contrast media.

This study analyzed the characteristics and differential points of spectral CT of metastatic and non-metastatic LNs in lung cancer patients. Different from the previous research, which focus on analyzing enhanced images, we carried out energy spectrum imaging on unenhanced scans and found differences in the IC, NIC and CT value on unenhanced scan. This work has its limitations. One limitation is that we did not analyze whether there is a structural difference between the central area of the LNs and the surrounding area. Also, the sample size of this study was relatively small. We expect to be able to derive more accurate guidelines with an increased sample size.

\section{Conclusions}

Quantitative parameters of spectral CT have a certain effectiveness for differential diagnosis between metastatic and non-metastatic LNs in lung cancer patients.

\section{Acknowledgments}

Funding: Natural Science Foundation of Guangdong Province, China (2019A1515011382); Science and Technology Planning Project of Guangzhou, Guangdong Province, China (201904010130); 2019 Achievement Transformation and Cultivation Project of the First Affiliated Hospital of Guangzhou Medical University (ZH201906).

\section{Footnote}

Reporting Checklist: The authors have completed the STROBE reporting checklist. Available at https://dx.doi. org/10.21037/jtd-21-385

Data Sharing Statement: Available at https://dx.doi. org/10.21037/jtd-21-385

Peer Review File: Available at https://dx.doi.org/10.21037/ jtd-21-385

Conflicts of Interest: All authors have completed the ICMJE uniform disclosure form (available at https://dx.doi. org/10.21037/jtd-21-385). The work of HC was supported by the Natural Science Foundation of Guangdong Province, China (2019A1515011382) and the Science and Technology Planning Project of Guangzhou, Guangdong Province, China (201904010130). The work of Suidan Huang was supported by 2019 Achievement Transformation and Cultivation Project of the First Affiliated Hospital of Guangzhou Medical University (ZH201906). The other authors have no conflicts of interest to declare.

Ethical Statement: The authors are accountable for all aspects of the work in ensuring that questions related to the accuracy or integrity of any part of the work are appropriately investigated and resolved. The study was conducted in accordance with the Declaration of Helsinki (as revised in 2013). The study was approved by the Ethics committee of First Affiliated Hospital of Guangzhou Medical University (Medical research ethical review 2019, No. K47) and individual consent for this retrospective analysis was waived.

Open Access Statement: This is an Open Access article 
distributed in accordance with the Creative Commons Attribution-NonCommercial-NoDerivs 4.0 International License (CC BY-NC-ND 4.0), which permits the noncommercial replication and distribution of the article with the strict proviso that no changes or edits are made and the original work is properly cited (including links to both the formal publication through the relevant DOI and the license). See: https://creativecommons.org/licenses/by-nc-nd/4.0/.

\section{References}

1. Nasim F, Sabath BF, Eapen GA. Lung Cancer. Med Clin North Am 2019;103:463-73.

2. Eberhardt WE, Mitchell A, Crowley J, et al. The IASLC Lung Cancer Staging Project: Proposals for the Revision of the $M$ Descriptors in the Forthcoming Eighth Edition of the TNM Classification of Lung Cancer. J Thorac Oncol 2015;10:1515-22.

3. Adam MA, Pura J, Goffredo P, et al. Presence and Number of Lymph Node Metastases Are Associated with Compromised Survival for Patients Younger Than Age 45 Years With Papillary Thyroid Cancer. J Clin Oncol 2015;33:2370-5.

4. Almeida FA, Uzbeck M, Ost D. Initial evaluation of the nonsmall cell lung cancer patient: diagnosis and staging. Curr Opin Pulm Med 2010;16:307-14.

5. Nguyen P, Bhatt M, Bashirzadeh F, et al. Comparison of objective criteria and expert visual interpretation to classify benign and malignant hilar and mediastinal nodes on $18-\mathrm{F}$ FDG PET/CT. Respirology 2015;20:129-37.

6. Baxa J, Vondráková A, Matoušková T, et al. Dual-phase dual-energy CT in patients with lung cancer: assessment of the additional value of iodine quantification in lymph node therapy response. Eur Radiol 2014;24:1981-8.

7. Zhao L, He ZY, Zhong XN, et al. (18)FDG-PET/CT for detection of mediastinal nodal metastasis in non-small cell lung cancer: a meta-analysis. Surg Oncol 2012;21:230-6.

8. Zhai X, Guo Y, Qian X. Combination of Fluorine-18 Fluorodeoxyglucose Positron-Emission Tomography/ Computed Tomography $\left({ }^{18} \mathrm{~F}-\mathrm{FDG}\right.$ PET/CT $)$ and Tumor Markers to Diagnose Lymph Node Metastasis in NonSmall Cell Lung Cancer (NSCLC): A Retrospective and Prospective Study. Med Sci Monit 2020;26:e922675.

9. Seol HY, Kim YS, Kim SJ. Predictive Value of 18F-Fluorodeoxyglucose Positron Emission Tomography or Positron Emission Tomography/Computed Tomography for Assessment of Occult Lymph Node Metastasis in Non-Small Cell Lung Cancer. Oncology
2021;99:96-104.

10. Yasufuku K, Pierre A, Darling G, et al. A prospective controlled trial of endobronchial ultrasound-guided transbronchial needle aspiration compared with mediastinoscopy for mediastinal lymph node staging of lung cancer. J Thorac Cardiovasc Surg 2011;142:1393400.e1.

11. Ohana M, Jeung MY, Labani A, et al. Thoracic dual energy CT: acquisition protocols, current applications and future developments. Diagn Interv Imaging 2014;95:1017-26.

12. Hurrell MA, Butler AP, Cook NJ, et al. Spectral Hounsfield units: a new radiological concept. Eur Radiol 2012;22:1008-13.

13. Krauss B. Dual-Energy Computed Tomography: Technology and Challenges. Radiol Clin North Am 2018;56:497-506.

14. Sun YS, Zhang XY, Cui Y, et al. Spectral CT imaging as a new quantitative tool? Assessment of perfusion defects of pulmonary parenchyma in patients with lung cancer. Chin J Cancer Res 2013;25:722-8.

15. Liu H, Yan F, Pan Z, et al. Evaluation of dual energy spectral CT in differentiating metastatic from nonmetastatic lymph nodes in rectal cancer: Initial experience. Eur J Radiol 2015;84:228-34.

16. Li J, Fang M, Wang R, et al. Diagnostic accuracy of dualenergy CT-based nomograms to predict lymph node metastasis in gastric cancer. Eur Radiol 2018;28:5241-9.

17. Liang H, Li A, Li Y, et al. A retrospective study of dualenergy CT for clinical detecting of metastatic cervical lymph nodes in laryngeal and hypopharyngeal squamous cell carcinoma. Acta Otolaryngol 2015;135:722-8.

18. Li X, Meng X, Ye Z. Iodine quantification to characterize primary lesions, metastatic and non-metastatic lymph nodes in lung cancers by dual energy computed tomography: An initial experience. Eur J Radiol 2016;85:1219-23.

19. Wu F, Zhou H, Li F, et al. Spectral CT Imaging of Lung Cancer: Quantitative Analysis of Spectral Parameters and Their Correlation with Tumor Characteristics. Acad Radiol 2018;25:1398-404.

20. Asamura H, Chansky K, Crowley J, et al. The International Association for the Study of Lung Cancer Lung Cancer Staging Project: Proposals for the Revision of the $\mathrm{N}$ Descriptors in the Forthcoming 8th Edition of the TNM Classification for Lung Cancer. J Thorac Oncol 2015;10:1675-84.

21. Goldstraw P, Chansky K, Crowley J, et al. The IASLC 
Lung Cancer Staging Project: Proposals for Revision of the TNM Stage Groupings in the Forthcoming (Eighth) Edition of the TNM Classification for Lung Cancer. J Thorac Oncol 2016;11:39-51.

22. Krantz SB, Lutfi W, Kuchta K, et al. Improved Lymph Node Staging in Early-Stage Lung Cancer in the National Cancer Database. Ann Thorac Surg 2017;104:1805-14.

23. Liang W, He J, Shen $Y$, et al. Impact of Examined Lymph Node Count on Precise Staging and Long-Term Survival of Resected Non-Small-Cell Lung Cancer: A Population Study of the US SEER Database and a Chinese MultiInstitutional Registry. J Clin Oncol 2017;35:1162-70.

24. Karçaaltıncaba M, Aktaş A. Dual-energy CT revisited with multidetector CT: review of principles and clinical applications. Diagn Interv Radiol 2011;17:181-94.

25. Apfaltrer P, Meyer M, Meier C, et al. Contrast-enhanced dual-energy CT of gastrointestinal stromal tumors: is iodine-related attenuation a potential indicator of tumor response? Invest Radiol 2012;47:65-70.

Cite this article as: Huang S, Meng H, Cen R, Ni Z, Li X, Suwal S, Chen H. Use quantitative parameters in spectral computed tomography for the differential diagnosis of metastatic mediastinal lymph nodes in lung cancer patients. J Thorac Dis 2021;13(8):4703-4713. doi: 10.21037/jtd-21-385
26. Chen X, Xu Y, Duan J, et al. Correlation of iodine uptake and perfusion parameters between dual-energy CT imaging and first-pass dual-input perfusion CT in lung cancer. Medicine (Baltimore) 2017;96:e7479.

27. Rizzo S, Radice D, Femia M, et al. Metastatic and nonmetastatic lymph nodes: quantification and different distribution of iodine uptake assessed by dual-energy CT. Eur Radiol 2018;28:760-9.

28. Kato T, Uehara K, Ishigaki S, et al. Clinical significance of dual-energy CT-derived iodine quantification in the diagnosis of metastatic LN in colorectal cancer. Eur J Surg Oncol 2015;41:1464-70.

29. Yazdani S, Miki Y, Tamaki K, et al. Proliferation and maturation of intratumoral blood vessels in non-small cell lung cancer. Hum Pathol 2013;44:1586-96.

30. Naresh KN, Nerurkar AY, Borges AM. Angiogenesis is redundant for tumour growth in lymph node metastases. Histopathology 2001;38:466-70. 
Supplementary

Table S1 Morphological and pathological results of LNs

\begin{tabular}{lccr}
\hline \multirow{2}{*}{ Pathology } & \multicolumn{2}{c}{ Short diameter of lymph node } & Total \\
\cline { 2 - 3 } & $\geq 10 \mathrm{~mm}$ & $<10 \mathrm{~mm}$ & $74(100.0)$ \\
Metastatic LNs, $\mathrm{n}(\%)$ & $48(64.9)$ & $26(35.1)$ & $152(100.0)$ \\
Non-metastatic LNs, $\mathrm{n}(\%)$ & $24(15.8)$ & $128(84.2)$ & 226 \\
Total & 72 & 154 & 226 \\
\hline
\end{tabular}

LNs, lymph nodes. 